\title{
A Low Complexity User Scheduling Algorithm for Uplink Multiuser MIMO Systems
}

\author{
Yangyang Zhang, Student Member, IEEE, Chunlin Ji, Yi Liu, Wasim Q. Malik, Member, IEEE, \\ Dominic C. O’Brien, and David J. Edwards
}

\begin{abstract}
A low complexity user scheduling algorithm based on a novel adaptive Markov chain Monte Carlo (AMCMC) method is proposed to achieve the maximal sum capacity in an uplink multiple-input multiple-output (MIMO) multiuser system. Compared with the existing scheduling algorithms, our algorithm is not only more efficient but also converges to within $99 \%$ of the optimal capacity obtained by exhaustive search. We demonstrate the convergence of the proposed scheduling algorithm and study the tradeoff between its complexity and performance.
\end{abstract}

Index Terms-Adaptive Markov chain Monte Carlo (AMCMC), multiple-input multiple-output (MIMO), multiuser selection, scheduling, sum capacity.

\section{INTRODUCTION}

$\mathbf{N}$ EXT generation wireless communication systems are expected to provide higher data rates to meet the increasing requirements of multimedia services. In order to utilize the spectrum efficiently, a technique known as multipleinput multiple-output (MIMO), has been extensively investigated [1]. MIMO wireless systems have been demonstrated to provide substantially higher link performance than traditional systems with the help of multiple antenna arrays. In multiuser systems, selection diversity plays an important role to improve the system performance, gauged in terms of the sum capacity and bit error rate (BER) [2]. To illustrate this, we consider a multiuser system, in which there is a base station (BS) with multiple receive antennas, and mobile users each with multiple transmit antennas, as shown in Fig. 1. A technique called user scheduling can be used to improve the system performance of multiuser systems while preserving the advantages of MIMO wireless systems. With the help of scheduling, the BS can optimally connect to the best subset of users at each time slot to maximize the sum capacity of the multiuser system. Recently, some research has been undertaken on efficient scheduling algorithms for multiuser systems. A scheduling algorithm based on system utility functions using a genetic algorithm (GA) was presented in [2] and a low complexity

Manuscript received February 16, 2007; revised July 16, 2007 and September 16, 2007; accepted September 25, 2007. The associate editor coordinating the review of this letter and approving it for publication was P. Martin. This work was supported in part by EPSRC Grant GR/T21769/01 and K. C. Wong Scholarship from the University of Oxford.

Y. Zhang, Y. Liu, D. C. O'Brien, and D. J. Edwards are with the Department of Engineering Science, University of Oxford, Parks Road Oxford OX1 3PJ, UK (e-mail: \{yangyang.zhang, y.liu, dominic.obrien, david.edwards\}@eng.ox.ac.uk).

C. Ji is with the Department of Statistical Science, Duke University, Durham, North Carolina 27708, USA (e-mail: chunlin.ji@duke.edu).

W. Q. Malik is with the Laboratory for Information and Decision Systems, Massachusetts Institute of Technology, 77 Massachusetts Avenue, Cambridge, MA 02139, USA (e-mail: wqm@mit.edu).

Digital Object Identifier 10.1109/TWC.2008.070199. user selection algorithm with block diagonalization (BD) was proposed in [3]. In this letter, we present a new low complexity user selection algorithm based on the adaptive Markov chain Monte Carlo (AMCMC) optimization technique [12] to maximize the sum capacity. The simulation results indicate that the proposed scheduling algorithm has a lower complexity order than the alternative approaches. Moreover the result lies within $99 \%$ of the optimal capacity obtained by exhaustive search.

\section{Uplink Multiuser MiMO System Model}

In Fig. 1, we consider an uplink multiuser MIMO system with a BS deploying $N_{R}$ receive antennas and $K$ mobile users with $N_{T}$ transmit antennas per user. The channel is assumed to be quasi-static fading [4]. Then the received signal at the base station is represented as [5]

$$
\mathbf{y}=\sum_{k=1}^{K} \mathbf{H}_{k} \mathbf{x}_{k}+\mathbf{v}
$$

where $\mathbf{y} \in \mathcal{C}^{N_{R} \times 1}$ is the received signal vector and $\mathbf{x}_{k} \in$ $\mathcal{C}^{N_{T} \times 1}$ is the transmitted signal vector for the $k^{\text {th }}$ mobile user with $\operatorname{Tr}\left(\mathbf{Q}_{k}\right) \leq P_{k}$ where $\mathbf{Q}_{k}=\mathbb{E}\left\{\mathbf{x}_{k} \mathbf{x}_{k}^{\mathrm{H}}\right\}$. Here, $\operatorname{Tr}(\cdot)$ stands for the matrix trace operation, $\mathbf{Q}_{k}$ is the signal covariance matrix, $P_{k}$ is the average power constraint for mobile user $k, \mathbb{E}\{\cdot\}$ denotes the statistical expectation and $(\cdot)^{\mathrm{H}}$ represents the Hermitian operation. The vector $\mathbf{v} \in \mathcal{C}^{N_{R} \times 1}$ is the independently identically distributed (i.i.d.) complex additive white Gaussian noise vector with distribution $\mathcal{C N}\left(0, N_{0} \mathbf{I}\right)$. The channel is described by an $N_{R} \times N_{T}$ complex random matrix, denoted by $\mathbf{H}_{k}$ whose entries, $\left[\mathbf{H}_{k}\right]_{(i, j)}\left(i=1 \ldots N_{R} ; j=1 \ldots N_{T}\right)$, represent the channel fading coefficient between the $i^{t h}$ receive antenna of BS and the $j^{\text {th }}$ transmit antenna of mobile user $k$. For the uncorrelated channels, the entries of $\mathbf{H}_{k}$ follow the i.i.d. complex Gaussian distribution $\mathcal{C N}(0,1)$. Moreover, it is assumed that perfect channel state information (CSI) is available at the receiver (i.e. the BS or access point).

The optimal sum capacity for the MIMO multiple-access channel is [5]

$$
C_{\text {sum }}\left(\mathbf{H}_{k}\right)=\max _{\sum_{i=1}^{K} \operatorname{Tr}\left(\mathbf{Q}_{k}\right) \leq P_{s}} \log _{2}\left|\mathbf{I}_{N_{R}}+\frac{1}{N_{0}} \sum_{k=1}^{K} \mathbf{H}_{k} \mathbf{Q}_{k} \mathbf{H}_{k}^{\mathrm{H}}\right|
$$

where $N_{0}$ is the average noise power. Note that (2) is the general capacity expression and not specialized to linear receivers. When CSI is not available to the transmitter, a reasonable transmission strategy is that the uniform power is distributed among the antennas of a user, $\mathbf{Q}_{k}=P_{k} \mathbf{I}_{N_{T}} / N_{T}$. Moreover, the total power constraint should be satisfied, $\sum_{k=1}^{K} P_{k}=P_{s}$, where $P_{s}$ is the total power of all mobile users. 


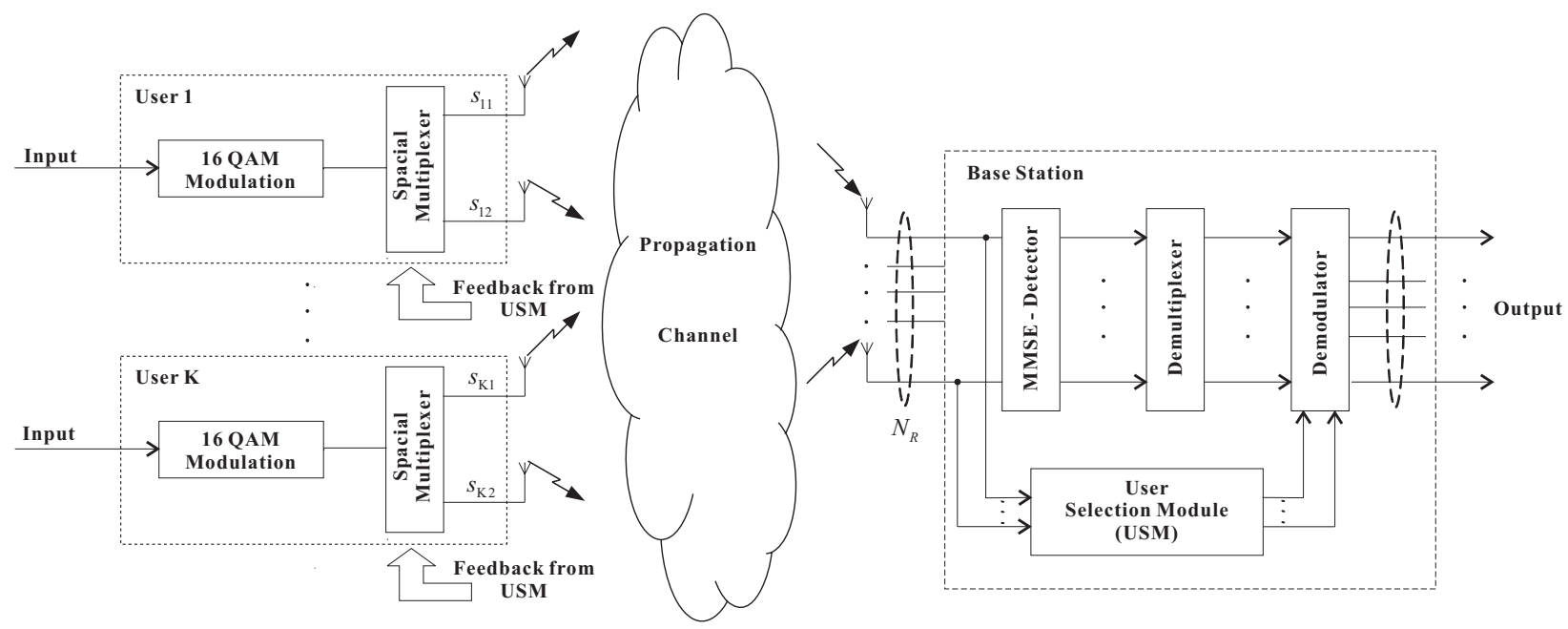

Fig. 1. Block diagram of the uplink multiuser MIMO system.

We denote the number of selected mobile users by $K_{\text {sel }}$. Considering a linear receiver, in order to detect all transmitted data streams at the BS, we have $\left(K_{\text {sel }} \times N_{T}\right) \leq N_{R}$ or, $K_{\text {sel }} \leq N_{R} / N_{T}$. Moreover, for uplink multiuser MIMO systems, a user selection module (USM) is centralized at the base station and the information of selected user indices is transmitted from base station to mobile users via an error-free and low-delay feedback channel. We further denote the set of all $\mathcal{Q}=\sum_{j=1}^{K_{\text {sel }}}\left(\begin{array}{c}K \\ j\end{array}\right)$ mobile user subset as $\boldsymbol{\Omega}=\left\{\boldsymbol{\omega}_{1}, \cdots, \boldsymbol{\omega}_{\mathcal{Q}}\right\}$ and the indicator of the selected subset of users by

$$
\boldsymbol{\omega}_{q}=\left\{I_{\alpha}\right\}_{\alpha=1}^{K}, I_{\alpha} \in\{0,1\}
$$

where $\alpha$ is the index of selected users and the indicator function $I_{\alpha}$ indicates whether the $\alpha^{t h}$ user is selected or not. For example, if the first, second, fifth and seventh users are selected, then $\boldsymbol{\omega}_{q}=\left\{1,1,0,0,1,0,1, \cdots, I_{K}\right\}$. According to (2), the sum capacity associated with the selection is then described as

$$
C_{\text {sum }}\left(\mathbf{H}_{\boldsymbol{\omega}_{q}}\right)=\max _{\boldsymbol{\omega}_{q} \in \boldsymbol{\Omega}} \log _{2}\left|\mathbf{I}_{N_{R}}+\frac{\rho}{N_{T}} \sum_{k=1}^{K} \mathbf{H}_{\left[\boldsymbol{\omega}_{q}\right]_{k}}\left(\mathbf{H}_{\left[\boldsymbol{\omega}_{q}\right]_{k}}\right)^{\mathrm{H}}\right|
$$

where $\rho=P_{k} / N_{0}$ is the average signal-to-noise ratio (SNR) and $\left[\boldsymbol{\omega}_{q}\right]_{k}$ denotes the $k^{t h}$ dimension of $\boldsymbol{\omega}_{q}$ and implies whether $k^{\text {th }}$ user is selected or not.

The most straightforward approach for solving (4) to obtain the optimal user subset, $\boldsymbol{\omega}^{*}$, is by an exhaustive search method, namely, finding all possible $\boldsymbol{\omega}_{q}$ out of $\boldsymbol{\Omega}$ to obtain the optimal selected user subset $\omega^{*}$ which can yield the maximum $C_{\text {sum }}\left(\mathbf{H}_{\boldsymbol{\omega}^{*}}\right)$. However, this method leads to a total of $\sum_{j=1}^{K_{s e l}}\left(\begin{array}{c}K \\ j\end{array}\right)$ possible combinations and becomes computationally expensive for the multiuser MIMO wireless systems with large $K$.

We now model (4) as the following combinatorial optimization problem

$$
\boldsymbol{\omega}^{*}=\arg \max _{\boldsymbol{\omega}_{q} \in \boldsymbol{\Omega}} C_{\text {sum }}\left(\mathbf{H}_{\boldsymbol{\omega}_{q}}\right)
$$

where $\boldsymbol{\omega}^{*}$ denotes the optimal selected user subset of the objective function, $C\left(\mathbf{H}_{\boldsymbol{\omega}_{q}}\right)$. An iterative optimization algo- rithm is proposed in the next section to solve the optimization problem described by (5).

\section{USER SELECTION ALGORITHM}

The Markov Chain Monte Carlo (MCMC) algorithm originated from [6], is a stochastic simulation technique designed to explore/sample a probability distribution of interest. It has gained tremendous popularity in the last few decades in a wide range of fields, such as engineering, statistics and biology [7]. It is computationally expensive, in general, to compare all the solutions to identify the optimal solution. So stochastic optimization/search methods have been developed with the idea that, instead of searching the whole solution space exhaustively, they just focus on exploring the 'promising' subspaces. To this end, MCMC is a powerful tool for stochastic optimization, given that one can appropriately represent the subspace of interest by a probability distribution [8]. Then, the samples $\left\{\boldsymbol{\omega}_{q}^{(n)}\right\}_{n=1}^{N}$ from an MCMC algorithm can also be used to estimate the maximum of the reformed objective function $\pi\left(\boldsymbol{\omega}_{q}\right)$ as follows

$$
\widehat{\boldsymbol{\omega}^{*}}=\arg \max _{\boldsymbol{\omega}_{q}^{(n)} ; n=1, \ldots, N} \pi\left(\boldsymbol{\omega}_{q}^{(n)}\right)
$$

where $\widehat{\omega^{*}}$ denotes the estimation of $\omega^{*}=$ $\arg \max _{\boldsymbol{\omega}_{q} \in \boldsymbol{\Omega}} \pi\left(\boldsymbol{\omega}_{q}\right)$.

To appropriately represent the feasible solution space by a probability distribution, one can use the Boltzmann distribution of the objective function $C_{\text {sum }}\left(\mathbf{H}_{\boldsymbol{\omega}_{q}}\right)$ with a proper chosen temperature $\tau: \pi\left(\boldsymbol{\omega}_{q}\right)=\exp \left\{\frac{C_{\text {sum }}\left(\mathbf{H}_{\omega_{q}}\right)}{\tau}\right\} / \Gamma$, where $\Gamma=\sum_{\boldsymbol{\omega}_{q} \in \boldsymbol{\Omega}} \exp \left\{\frac{C_{\text {sum }}\left(\mathbf{H}_{\boldsymbol{\omega}_{q}}\right)}{\tau}\right\}$ is a normalization constant, which can be ignored in the MCMC algorithm. Thus, maximizing $C_{\text {sum }}\left(\mathbf{H}_{\boldsymbol{\omega}_{q}}\right)$ is equivalent to maximizing $\pi\left(\boldsymbol{\omega}_{q}\right)$, i.e.,

$$
\boldsymbol{\omega}^{*}=\arg \max _{\boldsymbol{\omega}_{q} \in \boldsymbol{\Omega}} C_{\text {sum }}\left(\mathbf{H}_{\boldsymbol{\omega}_{q}}\right)=\arg \max _{\boldsymbol{\omega}_{q} \in \boldsymbol{\Omega}} \pi\left(\boldsymbol{\omega}_{q}\right)
$$

so $\widehat{\omega^{*}}$ from the MCMC algorithm is also the estimate of the maximum of $C_{\text {sum }}\left(\mathbf{H}_{\boldsymbol{\omega}_{q}}\right)$. 
The MCMC algorithm can be therefore applied to explore the distribution $\pi\left(\boldsymbol{\omega}_{q}\right)$. Here we take Metropolised independence sampler (MIS) [7], a generic MCMC algorithm, as an example. An initial value $\boldsymbol{\omega}_{q}^{(0)}$ is chosen for the algorithm. Given the current sample $\boldsymbol{\omega}_{q}^{(i)}$, a candidate sample $\boldsymbol{\omega}_{q}^{(\text {new })}$ is drawn from the proposal distribution $q\left(\boldsymbol{\omega}_{q} ; \mathbf{p}\right)$. According to the accepting probability $\left(\min \left\{1, \frac{\pi\left(\boldsymbol{\omega}_{q}^{(n e w)}\right)}{\pi\left(\boldsymbol{\omega}_{q}^{(i)}\right)} \frac{q\left(\boldsymbol{\omega}_{q}^{(i)}\right)}{q\left(\boldsymbol{\omega}_{q}^{(n e w)}\right)}\right\}\right)$, the new sample will be $\boldsymbol{\omega}_{q}^{(i+1)}=\boldsymbol{\omega}_{q}^{(\text {new })}$ if the $\boldsymbol{\omega}_{q}^{(\text {new })}$ is accepted, and $\boldsymbol{\omega}_{q}^{(i+1)}=\boldsymbol{\omega}_{q}^{(i)}$ otherwise. After $N$ iterations, we can obtain a set of samples $\left\{\boldsymbol{\omega}_{q}^{(0)}, \boldsymbol{\omega}_{q}^{(1)}, \boldsymbol{\omega}_{q}^{(2)}, \ldots \boldsymbol{\omega}_{q}^{(N)}\right\}$, which is subjected to the distribution $\pi\left(\boldsymbol{\omega}_{q}\right)$.

In traditional MCMC algorithms, for example the one mentioned above, adjusting the associated parameters $\mathbf{p}$ of the proposal density $q\left(\boldsymbol{\omega}_{q} ; \mathbf{p}\right)$ is crucial to achieve high convergence rate, but this process is not straightforward. Recently adaptive MCMC algorithms have been proposed to automatically adjust these parameters during simulations, and therefore improve the performance of MCMC in terms of both convergence and efficiency [13]. The user scheduling algorithm in this paper is based on the adaptive Markov Chain Monte Carlo (AMCMC) method proposed in [12]. The adaption strategy of the proposed AMCMC is described in the appendix, and a theorem is presented to guarantee the convergence of the proposed AMCMC algorithm. Here we give an analysis to demonstrate why the proposed AMCMC has fast convergence rate. For the sake of simplicity, we only focus on the MIS.

It is known that the performance of MIS is strongly dependent on the selection of the proposal density function. Its convergence rate is bounded by [7]

$$
\begin{aligned}
\left\|\mathcal{K}_{\mathbf{p}}^{n}\left(\boldsymbol{\omega}_{q}, \cdot\right)-\pi(\cdot)\right\| & =\sup _{Z \in \sigma(\boldsymbol{\Omega})}\left|\mathcal{K}_{\mathbf{p}}^{n}\left(\boldsymbol{\omega}_{q}, Z\right)-\pi(Z)\right| \\
& \leq 2\left(1-\frac{1}{\mathrm{~W}^{*}}\right)^{n}
\end{aligned}
$$

where $\mathcal{K}_{\mathbf{p}}^{n}\left(\boldsymbol{\omega}_{q}, \cdot\right)$ are the $n$-step transition probabilities with initial state $\boldsymbol{\omega}_{q}$ (see (15) in the appendix and [7]), $\sigma(\boldsymbol{\Omega})$ is the $\sigma$-field of the solution space $\Omega, Z$ is a subset in $\sigma(\boldsymbol{\Omega})$, and $\mathrm{W}^{*} \equiv \sup _{\boldsymbol{\omega}_{q} \in \sigma(\boldsymbol{\Omega})} \frac{\pi\left(\boldsymbol{\omega}_{q}\right)}{q\left(\boldsymbol{\omega}_{q} ; \mathbf{p}\right)}$. Note that, in contrast with other adaptive MCMC algorithms, the adaption strategy in our AMCMC algorithm is to minimize the KullbackLeibler divergence [9] between the distribution $\pi\left(\boldsymbol{\omega}_{q}\right)$ and the proposal distribution $q\left(\boldsymbol{\omega}_{q} ; \mathbf{p}\right)$

$$
\mathcal{D}\left[\pi\left(\boldsymbol{\omega}_{q}\right) \| q\left(\boldsymbol{\omega}_{q} ; \mathbf{p}\right)\right]=\sum_{q=1}^{\mathcal{Q}} \pi\left(\boldsymbol{\omega}_{q}\right) \times \log \left(\frac{\pi\left(\boldsymbol{\omega}_{q}\right)}{q\left(\boldsymbol{\omega}_{q} ; \mathbf{p}\right)}\right) .
$$

Intuitively, as the adaption runs iteratively, $\mathcal{D}\left[\pi\left(\boldsymbol{\omega}_{q}\right) \| q\left(\boldsymbol{\omega}_{q} ; \mathbf{p}\right)\right]$ becomes smaller, so does $\sup \frac{\pi\left(\boldsymbol{\omega}_{q}\right)}{q\left(\boldsymbol{\omega}_{q} ; \mathbf{p}\right)}$, and then the bound of convergence rate becomes smaller. In an ideal case, the adaption can make $\mathcal{D}\left[\pi\left(\boldsymbol{\omega}_{q}\right) \| q\left(\boldsymbol{\omega}_{q} ; \mathbf{p}\right)\right] \rightarrow 0$, and then the bound of convergence rate will also approach 0 even with a small value of $n$. This implies that starting with any initial value $\boldsymbol{\omega}_{q}$, the chain has the chance to jump into any space $Z \in \sigma(\boldsymbol{\Omega})$ with probability $\pi(Z)$ in a few steps. With the careful design of $\pi\left(\boldsymbol{\omega}_{q}\right)$ described above, the neighborhood of $\omega^{*}$ has a large probability mass, so the proposed AMCMC algorithm has a large chance to visit the neighborhood of $\omega^{*}$ and therefore can find at least a close-to-optimal solution $\widehat{\boldsymbol{\omega}^{*}}$ in a reasonable duration.

Due to the advantage of Monte Carlo method that the complexity of MCMC algorithm is dimension-independent and only related to the sample size $N$, and the fast convergence rate gained via AMCMC algorithm, we can get an efficient stochastic optimization algorithm with low complexity order. The AMCMC algorithm with MIS for user selection problem for uplink multiuser systems is described as follows:

Step 1: Initialize $\boldsymbol{\omega}_{q}^{(0)}$ randomly or deterministically, $\widehat{\boldsymbol{\omega}^{*}}=$ $\boldsymbol{\omega}_{q}^{(0)}$, and set $\mathbf{p}^{(0)}=\left\{p_{j}^{(0)}\right\}_{j=1}^{K}, p_{j}^{(0)}=\frac{1}{2}$ for the proposal density function $q\left(\boldsymbol{\omega}_{q}, \mathbf{p}^{(0)}\right)$. Set the iteration counter $t:=1$;

Step 2: Run the MIS, draw a small set of samples $\left\{\boldsymbol{\omega}_{q}^{(n)}\right\}_{n=1}^{N}$ from the objective function $\pi\left(\boldsymbol{\omega}_{q}\right)$ using the proposal $q\left(\boldsymbol{\omega}_{q}, \mathbf{p}^{(t-1)}\right)$;

Step 3: Update the parameter $p_{j}^{(t)}$ via

$$
p_{j}^{(t+1)}=p_{j}^{(t)}+r^{(t+1)}\left(\frac{1}{N} \sum_{n=1}^{N}\left[\boldsymbol{\omega}_{q}^{(n)}\right]_{j}-p_{j}^{(t)}\right)
$$

where the probability entries, $p_{j}, j=1, \cdots, K$, represent the probability of the $j^{t h}$ user to be chosen, $r^{(t)}$ is the sequence of decreasing step-sizes, and $\left[\boldsymbol{\omega}_{q}^{(n)}\right]_{j}$ represent the $j^{\text {th }}$ dimension of $\boldsymbol{\omega}_{q}^{(n)}$. Further details of (10) can be found in the appendix.

Step 4: If $\pi\left(\boldsymbol{\omega}_{q}^{(n)}\right)>\pi\left(\widehat{\boldsymbol{\omega}^{*}}\right)$ for $n=1, \cdots, N$, then $\widehat{\boldsymbol{\omega}^{*}}=$ $\boldsymbol{\omega}_{k}^{(n)}$.

Step 5: If the stopping criterion is satisfied, then stop; otherwise set $t:=t+1$ and go back to step 2. Here, the stopping criterion is the predefined number of iterations.

\section{Simulation Results}

Fig. 2 shows the average sum capacity averaged over 10,000 channel realizations versus the number of users, $K$, for $N_{T}=2, N_{R}=4$ and $K_{\text {sel }}=2$. It is seen that the performance obtained by the proposed AMCMC selection algorithm is nearly the same as the results obtained by exhaustive search. In fact, the simulation results indicate that the performance difference between these two selection schemes is within $1 \%$. Moreover, we find that the sum capacity increases as the number of users $(K)$ increases when the number of selected users $\left(K_{\text {sel }}\right)$ is constant. The reason for this gain is the multiuser diversity provided by the spatially distributed multiuser structure.

The average sum capacity versus SNR can be seen in Fig. 3 , from which we find that the AMCMC algorithm is not sensitive to the SNR. The situation is different for normbased selection (NBS) ${ }^{1}$ algorithm whose performance is nearly optimal in the low SNR (SNR $\leq 0 \mathrm{~dB})$ region but suboptimal at high SNR. Fig. 4 illustrates the BER versus SNR under various combination of $\left(K, K_{\text {sel }}\right)$ using the AMCMC algorithm. A 16QAM modulated system with a multi-user minimum meansquare error (MMSE) receiver is considered in this letter. The results validate the observation that selection diversity

\footnotetext{
${ }^{1}$ NBS is based on the channel Frobenius norm and indicates the power of the channel [3].
} 


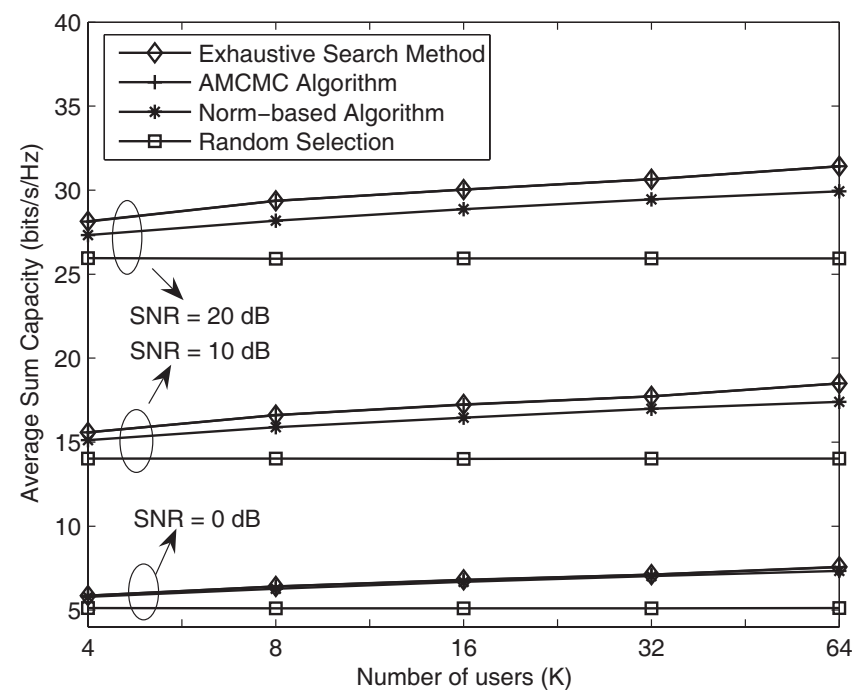

Fig. 2. Average sum capacity versus the number of users at $K_{\text {sel }}=2$ with $N_{R}=4, N_{T}=2$.

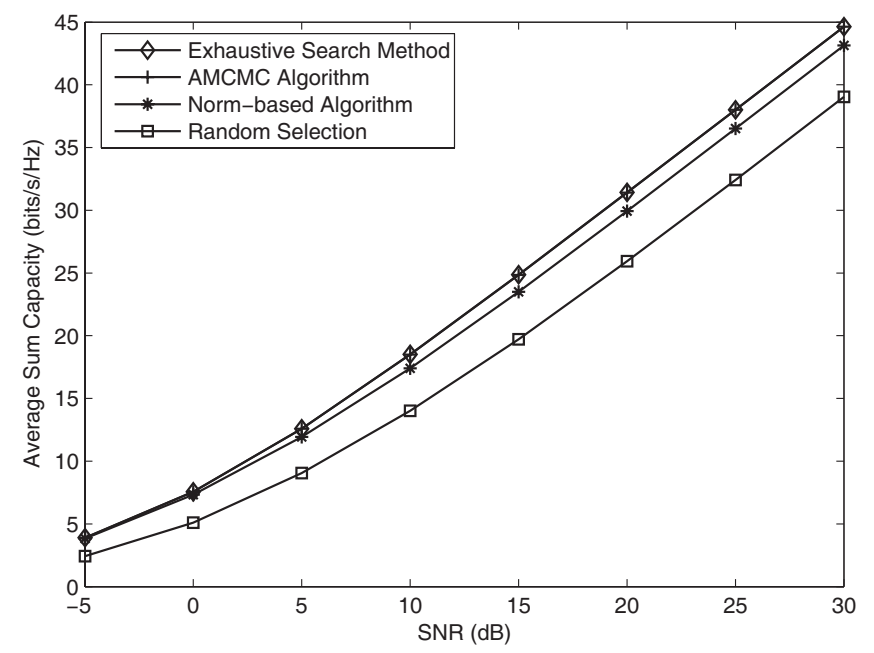

Fig. 3. Average sum capacity versus SNR at $K_{\text {sel }}=2$ and $K=64$ with $N_{R}=4, N_{T}=2$.

in a multiuser system can not only improve the average sum capacity, but also the BER. From Figs 2 and 3, we can draw a conclusion that, for fixed SNR, the larger the total number of users $K$, the higher is the average sum capacity. Meanwhile, large $K$ leads to low BER for fixed number of selected users, $K_{\text {sel }}$, which can be observed from Fig. 4.

Table 1 shows the complexity comparisons in terms of the number of function evaluations among different user selection algorithms, such as the GA algorithm [2], the BD algorithm [3], the AMCMC algorithm and an exhaustive search method. From the results, we find that the AMCMC algorithm has lower complexity and better performance than the GA algorithm. Compared with the BD algorithm, the AMCMC algorithm uses comparable complexity to obtain better results which are within $99 \%$ of the optimal capacity, while the results obtained by the BD algorithm are within $95 \%$ of the optimal capacity. If we relax our performance constraint from $99 \%$ to $95 \%$, the complexity of the AMCMC algorithm will be

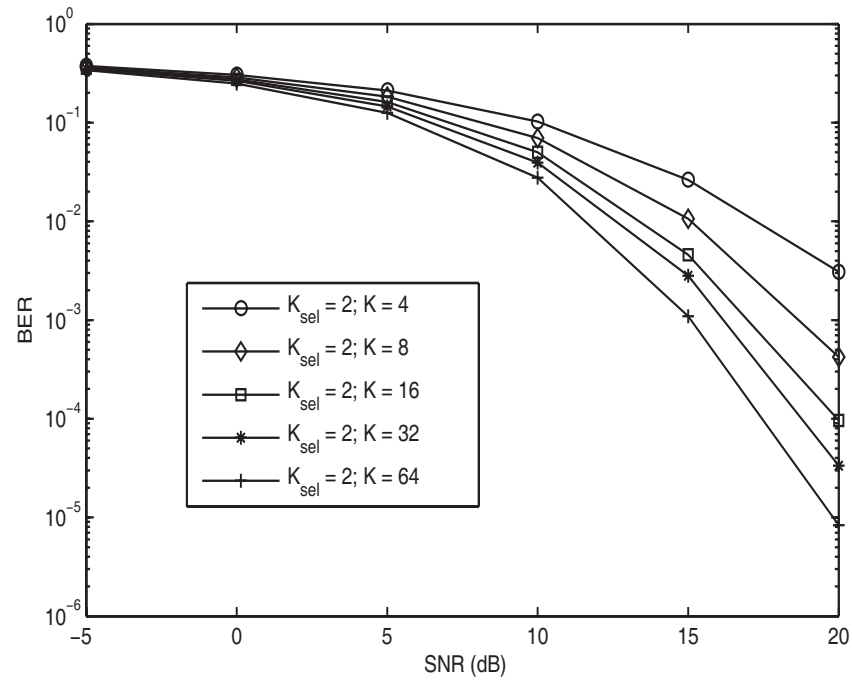

Fig. 4. System BER performance of choosing $K_{\text {sel }}$ out of $K$ at $N_{R}=4$, $N_{T}=2$ with AMCMC algorithm.

much lower than the BD algorithm. For example, in the case of choosing $K_{\text {sel }}=4$ out of $K=20$ users, the number of function evaluations of the AMCMC algorithm is 15 , but 80 for the BD algorithm.

Finally, the tradeoff between the complexity and performance of the AMCMC algorithm is addressed in Fig. 5. Because the AMCMC algorithm is an iterative algorithm, its complexity can be determined by the number of function evaluations, namely, $N \times t$, where $N$ is the number of samples drawn from $\pi\left(\boldsymbol{\omega}_{q}\right)$ for each iteration and $t$ is the number of iterations. Moreover we define the convergence ratio, $\vartheta=C_{A M C M C} / C_{\text {optimal }}$, to describe the performance of the AMCMC algorithm, where $C_{A M C M C}$ and $C_{\text {optimal }}$ denote the sum capacity obtained by AMCMC and an exhaustive search method, respectively. The relationship between the convergence ratio and the number of samples has been investigated in Fig. 5 for a fixed iteration number, $t=3$. In general, for the AMCMC algorithm, the higher the complexity (number of samples), the better the performance (convergence ratio). The feature of $\vartheta \rightarrow 1$ with smaller number of samples shows the efficiency of AMCMC algorithm.

\section{CONCLUSion}

In this letter, we have presented a novel user scheduling algorithm, based on adaptive Markov Chain Monte Carlo (AMCMC), to maximize the sum capacity of multiuser MIMO uplink systems. With the proposed algorithm, we can achieve results within $99 \%$ of the optimal capacity obtained by exhaustive search method. Moreover, we find that the proposed scheduling algorithm is reliable under a variety of SNR conditions. Finally, detailed complexity comparisons indicate that our user scheduling algorithm has much lower complexity and is more robust than other user scheduling algorithms. Thus it can make practical low-complexity uplink multiuser MIMO wireless communication systems easier to implement. 
TABLE I

PERFORMANCE AND COMPLEXITY COMPARISONS FOR VARIOUS USER SELECTION ALGORITHMS WITH $N_{R}=4, N_{T}=2, \rho=10$ DB AND $\vartheta=C / C_{\text {optimal }}$, IS THE RATIO OF THE AVERAGE SUM CAPACITY, $C$, OBTAINED BY A GIVEN ALGORITHM TO THAT OF THE OPTIMAL USER SELECTION ALGORITHM. NOTE THAT THE TWO AMCMC SCHEMES DESCRIBED BELOW ARE BASED ON THE SAME ALGORITHM BUT WITH DIFFERENT PERFORMANCE CRITERIA $(\vartheta)$.

\begin{tabular}{c|c|c|c|c|c}
\hline$\left(K, K_{\text {sel }}\right)$ & GA Algorithm [2] & BD Algorithm [3] & AMCMC Algorithm & AMCMC Algorithm & Exhaustive Search Method \\
\hline \hline$(10,2)$ & 30 & 20 & 12 & 24 & 55 \\
$(10,4)$ & 50 & 40 & 15 & 30 & 385 \\
$(20,2)$ & 60 & 40 & 15 & 45 & 210 \\
$(20,4)$ & 100 & $\geq 0$ & 15 & 60 & 6195 \\
\hline \hline$\vartheta$ & $\geq 90 \%$ & $\geq 95 \%$ & $\geq 99 \%$ & $=1$ \\
\hline
\end{tabular}

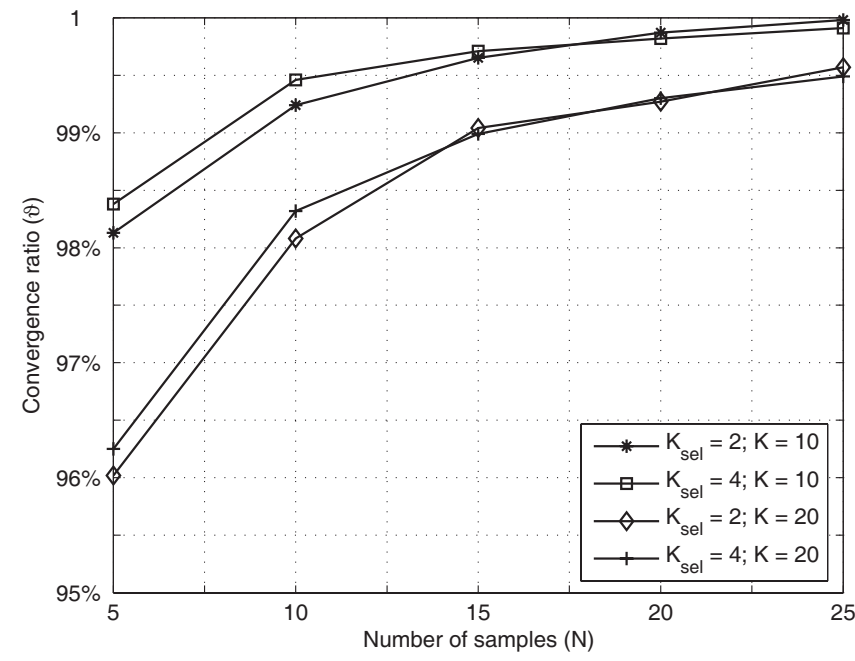

Fig. 5. Convergence ratio versus number of samples with three iterations, with $N_{R}=4, N_{T}=2$ and $\rho=10 \mathrm{~dB}$, using AMCMC algorithm.

\section{APPENDIX}

\section{Derivation: Updating RUle FOR AdAPTIVE MCMC} METHOD

In the AMCMC algorithm, the adaptive proposal distribution is proportional to the product of Bernoulli distributions, $q\left(\boldsymbol{\omega}_{q} ; \mathbf{p}\right)=\frac{\prod_{i=1}^{K} p_{i}^{\left[\boldsymbol{\omega}_{q}\right]_{i}}\left(1-p_{i}\right)^{1-\left[\boldsymbol{\omega}_{q}\right]_{i}}}{\Gamma^{\prime}} \propto \prod_{i=1}^{K} p_{i}^{\left[\boldsymbol{\omega}_{q}\right]_{i}}(1-$ $\left.p_{i}\right)^{1-\left[\boldsymbol{\omega}_{q}\right]_{i}}$, where $\boldsymbol{\omega}_{q}$ is the indicator of the selected subset of users and $\mathbf{p}=\left(p_{1}, \cdots, p_{K}\right)$ is the probability vector to indicate the probability of the users to be chosen. Specifically, $p_{i}$ denotes the probability of the $i^{t h}$ user to be chosen, $\left[\boldsymbol{\omega}_{q}\right]_{i}$ represents the $i^{\text {th }}$ dimension of $\boldsymbol{\omega}_{q}$ and implies whether $i^{\text {th }}$ user is selected or not, and $\Gamma^{\prime}=\sum_{q=1}^{\mathcal{Q}} \prod_{i=1}^{K} p_{i}^{\left[\boldsymbol{\omega}_{q}\right]_{i}}(1-$ $\left.p_{i}\right)^{1-\left[\boldsymbol{\omega}_{q}\right]_{i}}$ is a normalization constant, which can be ignored with no effect on the performance of the AMCMC algorithm. Note that in the proposal distribution, we assume that each $\left[\boldsymbol{\omega}_{q}\right]_{i}$ is independent, which implies that the event that the $i^{t h}$ user is selected is independent of an event in which any other user is selected. This is a reasonable assumption when the samples $\left[\boldsymbol{\omega}_{q}\right]_{i}$ in $\pi\left(\boldsymbol{\omega}_{q}\right)$ are not highly correlated.

The strategy is to adaptively tune the parameterized proposal distribution $q\left(\boldsymbol{\omega}_{q} ; \mathbf{p}\right)$ to approximate the target distribution $\pi\left(\boldsymbol{\omega}_{q}\right)$ in the sense of minimizing the KullbackLeibler divergence [9] between the distribution $\pi\left(\boldsymbol{\omega}_{q}\right)$ and the proposal distribution $q\left(\boldsymbol{\omega}_{q} ; \mathbf{p}\right)$

$$
\mathcal{D}\left[\pi\left(\boldsymbol{\omega}_{q}\right) \| q\left(\boldsymbol{\omega}_{q} ; \mathbf{p}\right)\right]=\sum_{q=1}^{\mathcal{Q}} \pi\left(\boldsymbol{\omega}_{q}\right) \times \log \left(\frac{\pi\left(\boldsymbol{\omega}_{q}\right)}{q\left(\boldsymbol{\omega}_{q} ; \mathbf{p}\right)}\right)
$$

where $\mathcal{Q}$ is the number of all the feasible $\boldsymbol{\omega}_{q}$. Since it is easy to prove that $\widetilde{\mathcal{D}}=\pi\left(\boldsymbol{\omega}_{q}\right) \times \log \pi\left(\boldsymbol{\omega}_{q}\right)-\mathcal{D}\left[\pi\left(\boldsymbol{\omega}_{q}\right) \| q\left(\boldsymbol{\omega}_{q} ; \mathbf{p}\right)\right]$ is a convex function [10], then to minimize the Kullback-Leibler divergence $\mathcal{D}\left[\pi\left(\boldsymbol{\omega}_{q}\right) \| q\left(\boldsymbol{\omega}_{q} ; \mathbf{p}\right)\right]$ w. r. t. $\mathbf{p}$ is equal to finding the root of $\partial \widetilde{\mathcal{D}} / \partial \mathbf{p}=0$. For each $p_{j}, j=1, \ldots, K$, we have

$$
\begin{aligned}
\frac{\partial \widetilde{\mathcal{D}}}{\partial p_{j}} & =\frac{\partial}{\partial p_{j}} \sum_{q=1}^{\mathcal{Q}} \pi\left(\boldsymbol{\omega}_{q}\right) \times \log q\left(\boldsymbol{\omega}_{q} ; \mathbf{p}\right) \\
& \propto \frac{\partial}{\partial p_{j}} \sum_{q=1}^{\mathcal{Q}} \pi\left(\boldsymbol{\omega}_{q}\right) \times \log \left(\prod_{i=1}^{K} p_{i}^{\boldsymbol{\omega}_{q}}\left(1-p_{i}\right)^{1-\boldsymbol{\omega}_{q}}\right) \\
& =\sum_{q=1}^{\mathcal{Q}} \pi\left(\left[\boldsymbol{\omega}_{q}\right]_{j}\right) \times\left(\frac{\left[\boldsymbol{\omega}_{q}\right]_{j}}{p_{j}}-\frac{1-\left[\boldsymbol{\omega}_{q}\right]_{j}}{1-p_{j}}\right) \\
& =\frac{1}{p_{j}\left(1-p_{j}\right)} \sum_{q=1}^{\mathcal{Q}} \pi\left(\left[\boldsymbol{\omega}_{q}\right]_{j}\right)\left(\left[\boldsymbol{\omega}_{q}\right]_{j}-p_{j}\right) .
\end{aligned}
$$

Generally $\pi\left(\boldsymbol{\omega}_{q}\right)$ or $\pi\left(\left[\boldsymbol{\omega}_{q}\right]_{j}\right)$ is unknown and it is inefficient and unnecessary to exhaustively enumerate all $\boldsymbol{\omega}_{q}$ to calculate $\partial \widetilde{\mathcal{D}} / \partial \mathbf{p}$. A feasible way is to calculate the Monte Carlo estimate of $\partial \widetilde{\mathcal{D}} / \partial \mathbf{p}$ and then use stochastic approximation techniques to find the root of $\partial \widetilde{\mathcal{D}} / \partial \mathbf{p}=0$ iteratively. Assume a set of samples $\left\{\boldsymbol{\omega}_{q}^{(n)}\right\}_{n=1}^{N}$ is drawn from $\pi\left(\boldsymbol{\omega}_{q}\right)$, then the Monte Carlo estimate of $\partial \widetilde{\mathcal{D}} / \partial \mathbf{p}$ is $\frac{1}{N}\left[\frac{1}{p_{j}\left(1-p_{j}\right)} \sum_{n=1}^{N} \sum_{q=1}^{\mathcal{Q}}\left(\left[\boldsymbol{\omega}_{q}^{(n)}\right]_{j}-p_{j}\right)\right]$. Employing the Robbins-Monro stochastic approximation algorithm [10], we can obtain the recursive update equation to approach the root of $\partial \widetilde{\mathcal{D}} / \partial \mathbf{p}=0$ :

$$
p_{j}^{(t+1)}=p_{j}^{(t)}+\frac{r^{(t+1)}}{p_{j}(t)\left(1-p_{j}^{(t)}\right)}\left(\frac{1}{N} \sum_{n=1}^{N}\left[\boldsymbol{\omega}_{q}^{(n)}\right]_{j}-p_{j}^{(t)}\right)
$$

where $r^{(t)}$ is a sequence of decreasing step-sizes, e.g. satisfying the conditions of $\sum_{t=0}^{\infty} r^{(t)}=\infty$ and $\sum_{t=0}^{\infty}\left(r^{(t)}\right)^{2}<\infty$ [11]. Since $p_{j}\left(1-p_{j}\right)$ has no significant influence on the convergence of (13), it can be simplified as

$$
p_{j}^{(t+1)}=p_{j}^{(t)}+r^{(t+1)}\left(\frac{1}{N} \sum_{n=1}^{N}\left[\boldsymbol{\omega}_{q}^{(n)}\right]_{j}-p_{j}^{(t)}\right) .
$$

With the proposed adaptive strategy, the algorithm actually runs a non-Markov chain because the proposal density is changed based on history information, and the convergence of 
this kind of algorithm is a major research issue. The ergodicity and convergence of a wide range of adaptive MCMC methods have been proven recently in [13]. Here we present a theorem to verify the ergodicity of an adaptive MCMC algorithm, which is easy to use in practice.

Theorem We now provide the proof of the convergence for our algorithm using the following adaptation of the theorem presented in [13]. Consider an adaptive MCMC algorithm, on a state space $\mathcal{F}=\sigma(\boldsymbol{\Omega})$. The $\pi(\cdot)$ is a stationary distribution for each transition kernel of MIS algorithm

$$
\mathcal{K}_{\mathbf{p}}\left(\boldsymbol{\omega}_{q}, \boldsymbol{\omega}_{q}^{\prime}\right)=\left\{\begin{array}{cc}
q\left(\boldsymbol{\omega}_{q}^{\prime} ; \mathbf{p}\right) W_{\min } & \text { if } \boldsymbol{\omega}_{q}^{\prime} \neq \boldsymbol{\omega}_{q} \\
\sum_{\boldsymbol{\omega}_{q}^{(j)} \neq \boldsymbol{\omega}_{q}} q\left(\boldsymbol{\omega}_{q}^{(j)} ; \mathbf{p}\right) W_{\max } & \\
+q\left(\boldsymbol{\omega}_{q} ; \mathbf{p}\right), & \text { otherwise }
\end{array}\right.
$$

where $W_{\boldsymbol{\omega}_{q}}=\pi\left(\boldsymbol{\omega}_{q}\right) / q\left(\boldsymbol{\omega}_{q} ; \mathbf{p}\right)$ is defined as the importance ratio, $W_{\min }$ represents $\min \left(1, \frac{W_{\boldsymbol{\omega}_{q}^{\prime}}}{W_{\boldsymbol{\omega}_{q}}}\right)$ and $W_{\max }$ denotes $\max \left(0,1-\frac{W_{\boldsymbol{\omega}_{q}^{(j)}}}{W_{\boldsymbol{\omega}_{q}}}\right)$. Assume that:

(a) [Simultaneous Uniform Ergodictiy]

For all $\epsilon>0$, there is $T=T(\epsilon) \in \mathcal{N}$ such that $\left\|\mathcal{K}_{\mathbf{p}}^{T}\left(\boldsymbol{\omega}_{q}, \cdot\right)-\pi(\cdot)\right\| \leq \epsilon$ for all $\boldsymbol{\omega}_{q}$ and $\mathbf{p}$;

(b) [Diminishing Adaption]

$\lim _{t \rightarrow \infty} \sup _{\boldsymbol{\omega}_{q} \in \mathcal{F}}\left\|\mathcal{K}_{\mathbf{p}^{(t+1)}}\left(\boldsymbol{\omega}_{q}, \cdot\right)-\mathcal{K}_{\mathbf{p}^{(t)}}\left(\boldsymbol{\omega}_{q}, \cdot\right)\right\|=0$ in probability.

Then the adaptive algorithm is ergodic, namely

$$
\lim _{t \rightarrow \infty} \sup _{Z \in \boldsymbol{\Omega}}\left|A^{(t)}\left(\left(\boldsymbol{\omega}_{q}, \mathbf{p}\right), Z\right)-\pi(Z)\right|
$$

where $A^{(t)}\left(\left(\boldsymbol{\omega}_{q}, \mathbf{p}\right), Z\right)=\operatorname{Pr}\left[\boldsymbol{\omega}_{q}^{(t)} \in Z \mid \boldsymbol{\omega}_{q}^{(0)}=\boldsymbol{\omega}_{q}, \mathbf{p}^{(0)}=\mathbf{p}\right]$ is the transition probability for $\boldsymbol{\omega}_{q}^{(t)}$ for the adaptive algorithm, given the initial conditions $\boldsymbol{\omega}_{q}^{(0)}=\boldsymbol{\omega}_{q}$ and $\mathbf{p}^{(0)}=\mathbf{p}$.

To satisfy the simultaneous uniform ergodictiy condition, we should restrict that $\mathbf{p}$ to be positive; to guarantee the Diminishing Adaption condition, we need to ensure $r^{(t)} \rightarrow 0$ as $t \rightarrow \infty$, which is consistent with the condition of $r^{(t)}$ in the stochastic approximation algorithm. With careful design of the
AMCMC algorithm, these two conditions can be satisfied and therefore the ergodicity of the algorithm holds, which implies that starting with any initial value, the algorithm can visit any space $Z \in \Omega$ with the probability $\pi(Z)$ in a sufficiently long duration. With careful design of $\pi\left(\boldsymbol{\omega}_{q}\right)$, the neighborhood of global optimum $\boldsymbol{\omega}^{*}$ has a large probability mass, so the algorithm has a good chance to visit the neighborhood of $\boldsymbol{\omega}^{*}$ and find at least a close-to-optimal solution in a reasonable time, and thus the AMCMC can be used as a generalized stochastic optimization tool.

\section{REFERENCES}

[1] E. Telatar, "Capacity of multi-antenna Gaussian channels," Eur. Trans. Telecommun., vol. 10, pp. 585-595, Nov. 1999.

[2] K. Lau, "Analytical framework for multiuser uplink MIMO space-time scheduling design with convex utility functions," IEEE Trans. Wireless Commun., vol. 3, no. 5, pp. 1832-1843, Sept. 2004.

[3] Z. Shen, R. Chen, J. Andrews, R. Heath, and B. Evans, "Low complexity user selection algorithm for multiuser MIMO systems with block diagonalization," IEEE Trans. Signal Processing, vol. 54, no. 9, pp. 3658-3663, 2006.

[4] D. Tse and P. Viswanath, Fundamentals of Wireless Communication. Cambridge University Press, 2005.

[5] S. Vishwanath, N. Jindal, and A. Goldsmith, "Duality, achievable rates, and sum-rate capacity of Gaussian MIMO broadcast channels," IEEE Trans. Inform. Theory, vol. 49, pp. 2658-2668, Oct. 2003.

[6] N. Metropolis, A. Rosenbluth, M. Rosenbluth, A. Teller, and E. Teller, "Equation of state calculations by fast computing machines," J. Chem. Phys., vol. 21, pp. 1087-1092, 1953.

[7] J. Liu, Monte Carlo Strategies in Scientific Computing. New York: Springer, 2001.

[8] C. Andrieu, N. de Freitas, A. Doucet, and M. I. Jordan, "An introduction to MCMC for machine learning," Machine Learning, vol. 50, pp. 5-43, 2003.

[9] S. Kullback and R. Leibler, "On information and sufficiency," Ann. Math. Stat., vol. 22, pp. 79-86, 1951.

[10] H. Robbins and S. Monro, "A stochastic approximation method," Ann. Math. Stat., vol. 22, pp. 400-407, 1951.

[11] H. Kushner and G. Yin, Stochastic Approximation Algorithms and Applications, 2nd edition. Springer-Verlag, 2003.

[12] C. Ji, "Adaptive Monte Carlo methods for Bayesian inference," MPhil thesis, Department of Engineering, University of Cambridge, UK, 2006.

[13] J. S. Rosenthal and G. O. Roberts, "Coupling and ergodicity of adaptive MCMC," J. Applied Probability, vol. 44, pp. 458-475, 2007. 